\title{
A Flat Faint End of the Fornax Cluster Galaxy Luminosity Function
}

\author{
S. Mieske ${ }^{1}$, M. Hilker ${ }^{1}$, L. Infante ${ }^{2}$ and C. Mendes de Oliveira ${ }^{3}$ \\ ${ }^{1}$ European Southern Observatory, Karl-Schwarzschild-Str.2, 85748 \\ Garching b. München, Germany \\ email: smieske@eso.org,mhilker@eso.org \\ ${ }^{2}$ Departamento de Astronomía y Astrofísica, Pontificia Universidad Católica \\ de Chile, Casilla 306, Santiago 22, Chile \\ email: linfante@astro.puc.cl \\ ${ }^{3}$ Instituto de Astronomia, Geofísica, e Ciências Atmosféricas, Departamento de Astronomia, \\ Universidade de São Paulo, Rua do Matáo 1226, Cidade Universitãria, \\ 05508-900 São Paulo, SP, Brazil \\ email: oliveira@astro.iag.usp.br
}

\begin{abstract}
We analyse the photometric properties of the early-type Fornax cluster dwarf galaxy population $\left(M_{V}>-17 \mathrm{mag}\right)$, based on a wide field imaging study of the central cluster area in $V$ and $I$ band-passes with IMACS/Magellan at Las Campanas Observatory. We create a fiducial sample of $\sim 100$ Fornax cluster dwarf ellipticals (dEs) with $-16.6<M_{V}<-8.8$ mag in the following three steps: (1) To verify cluster membership, we measured $I$-band surface brightness fluctuations (SBF) distances to candidate dEs known from previous surveys; (2) We re-assessed morphological classifications for those candidate dEs that are too faint for SBF detection; and (3) We searched for new candidate dEs in the size-luminosity regime close to the resolution limit of previous surveys. The resulting fiducial $\mathrm{dE}$ sample follows a well-defined surface brightness magnitude relation, showing that Fornax dEs are about $40 \%$ larger than Local Group dEs. The sample also defines a colour-magnitude relation similar to that of Local Group dEs. The earlytype dwarf galaxy luminosity function in Fornax has a very flat faint end slope $\alpha \simeq-1.1 \pm 0.1$. We compare the number of dwarfs per unit mass with those in other environments and find that the Fornax cluster fits well into a general trend of a lack of high-mass dwarfs in more massive environments.
\end{abstract}

Keywords. galaxies: clusters: individual: Fornax cluster - galaxies: dwarf - galaxies: fundamental parameters - galaxies: luminosity function -techniques: photometric

\section{Introduction}

One of the most important quantities for characterising a galaxy population is the galaxy luminosity function (GLF). Its logarithmic faint-end slope $\alpha$ is a very useful quantity to be contrasted with the expected slope for the mass spectrum of cosmological dark-matter halos (e.g. Jenkins et al. 2001, Moore et al. 1999). Generally, the value of $\alpha$ derived in various environments including the Local Group is much shallower than the expected slope of dark matter halos (see for example Grebel et al. 2003; Trentham \& Tully 2002; Trentham et al. 2005; Andreon et al. 2006; Tanaka et al. 2005; and Infante et al. 2003 and references therein). This discrepancy is also known as the "substructure problem" of present-day cosmology.

In this contribution we focus on the GLF in the nearby Fornax cluster of galaxies. Up to now, investigations of the Fornax GLF in the low luminosity regime $\left(M_{\mathrm{V}}>-14 \mathrm{mag}\right)$ have been restricted to morphological cluster-membership assignment, given the lacking depth of spectroscopic surveys. The important restriction of the morphological assessment 

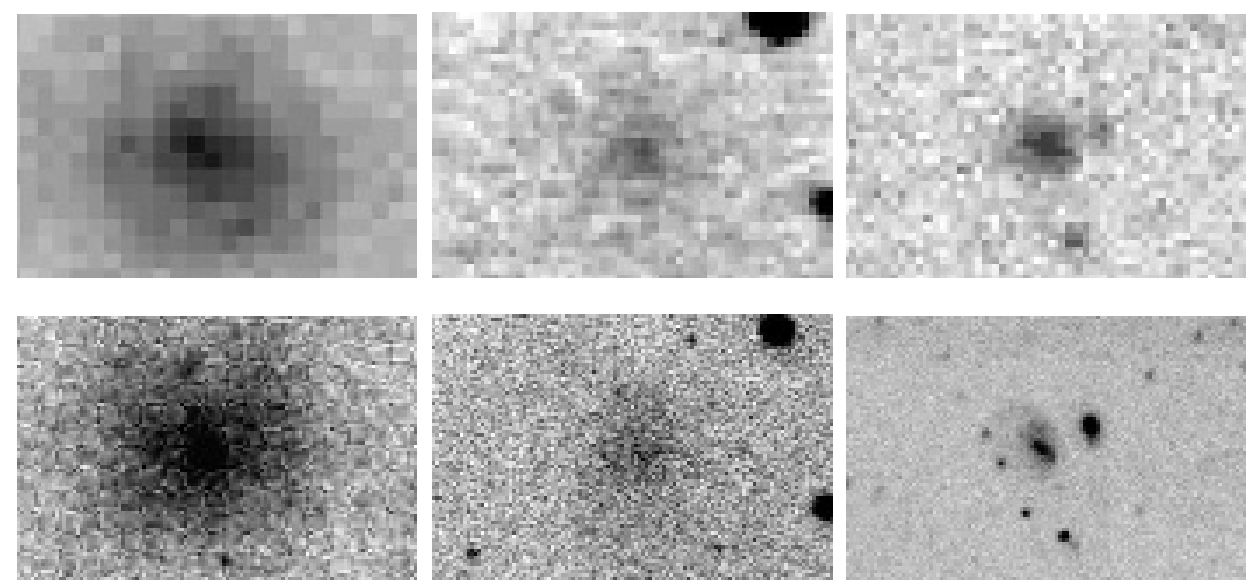

Figure 1. Top: Example thumbnails of three dwarf galaxy candidates from the sample of Hilker et al. (2003). Bottom: The same three galaxies, imaged with the higher resolution IMACS data (Mieske et al. 2007). Galaxies are from left to right: FCC 191, which is confirmed via SBF measurement with the higher resolution IMACS data; WFLSB2-1, whose morphological assessment as probable cluster member from Hilker et al. (2003) is confirmed with the IMACS data; WFLSB6-3, whose morphological assessment as probable cluster member from Hilker et al. (2003) is changed to probable background.

is the uncertainty in estimating the amount of contamination by background galaxies (e.g. Trentham \& Tully 2002). This can lead to different authors deriving very different slopes for the same cluster: Ferguson \& Sandage (1988) obtain $\alpha=-1.08 \pm 0.09$ for the dwarf GLF in Fornax; Kambas et al. (2000) suggest a much steeper slope $\alpha \simeq-2.0$, based on poorer resolution data of $2.3^{\prime \prime}$ without colour information (see also the discussion in Hilker et al. 2003). Such differences in $\alpha$ stress the need for high-resolution imaging and an extension of the limiting magnitude for direct cluster membership determination.

In this contribution (see also Mieske et al. 2007), we investigate the photometric properties of the dwarf galaxy population in the central 1.5 degree of the Fornax cluster $\left(M_{\mathrm{V}}>-17 \mathrm{mag}\right)$. For this we have used the wide-field imaging instrument IMACS mounted at the $6.5 \mathrm{~m}$ Magellan telescopes at LCO, yielding a $0.2^{\prime \prime}$ pixel scale. This work follows up a previous study with the $2.5 \mathrm{~m}$ du Pont telescope and the WFCCD camera (Hilker et al. 2003, based on $0.8^{\prime \prime}$ pixels. To overcome the difficulties of morphological cluster membership assignment, we adopt the following three steps to define a fiducial sample of Fornax cluster dwarf ellipticals.

1. Confirm cluster membership of candidate dEs via measuring surface brightness fluctuations (SBF, Tonry \& Schneider 1988), and hence their distance.

2. Re-assess the morphological classification of candidate dEs too faint for SBF.

3. Search for new candidate dEs close to the resolution limit of previous surveys.

The details of these procedures are described in Mieske et al. (2007). Fig. 1 gives example thumbnails of dwarf galaxy candidates, showing images from both the lower resolution WFCCD data and the higher resolution IMACS data.

\section{Results}

We confirm cluster membership for 28 candidate dEs in the range $-16.6<M_{V}<$ -10.1 mag by means of SBF measurement. Of 51 further candidate dEs in the range $-13.2<M_{V}<-8.6 \mathrm{mag}, 2 / 3$ are confirmed as probable cluster members by 

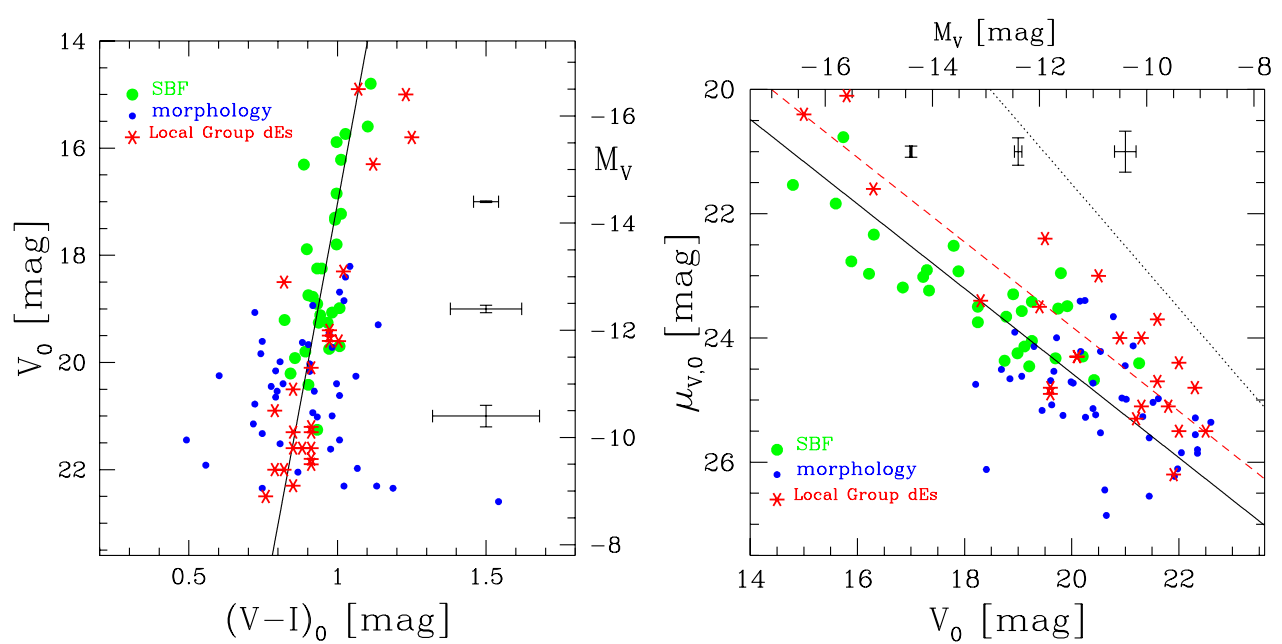

Figure 2. Colour-magnitude diagram (left) and surface brightness-magnitude diagram (right) of Fornax cluster early type dwarf galaxies. Large filled circles indicate dwarfs for which direct cluster membership via SBF measurement was obtained. Small filled circles indicate dwarfs for which the cluster membership assignment was via morphology. Asterisks indicate Local Group Dwarfs (Grebel et al. 2003). In both plots, the solid lines are fits to the Fornax data points. In the right plot, the dotted line indicates the resolution limit of our data. The dashed line is a fit to the Local Group data points.

morphological re-assessment, while $1 / 3$ are re-classified as probable background objects. Finally, we find 12 new $\mathrm{dE}$ candidates in the range $-12.3<M_{V}<-8.8 \mathrm{mag}$.

In Fig. 2 we show the colour-magnitude and surface brightness-magnitude relation of the resulting fiducial sample of Fornax dwarf elliptical galaxies. The colours of the Fornax dwarfs are very similar to their Local Group counterparts. Only the brightest Local Group dwarfs appear slightly redder (more metal-rich). The sizes of Fornax dwarfs are systematically larger than those in the Local Group by about 40\%. This higher compactness in the Local Group may have favoured self-enrichment more than in Fornax, possibly explaining the metallicity offset of the brightest Local Group dwarfs.

Fig. 3 shows the incompleteness corrected GLF of the sample. The derived faint end slope is $\alpha=-1.1 \pm 0.1$. Our results thus confirm a very shallow faint end slope for the Fornax dwarf galaxy luminosity function, in agreement with early estimates in the reference study of Ferguson \& Sandage (1988). We are therefore confident that this value is robust and not very biased by systematic effects. Morphological cluster membership assignment in Fornax apparently is very reliable, provided that the image resolution is sufficient.

\section{Discussion and conclusions}

It is well known that such a shallow faint end slope sharply contradicts the much steeper value predicted for the mass function of $\Lambda$ CDM halos (e.g.Kauffman et al. 2000, Moore et al. 1999). Possible reasons for that discrepancy include the accretion scenario (e.g. Hilker et al. 1999b, Côté et al. 1998), where dwarf galaxies that fall into the cluster centres are tidally disrupted, hence contributing to forming the extended $\mathrm{cD}$ halos of the most massive cluster galaxies like NGC 1399 in Fornax. The presence of ultra-compact dwarf galaxies (UCDs) in the central Fornax cluster (Hilker et al. 1999a, Drinkwater 


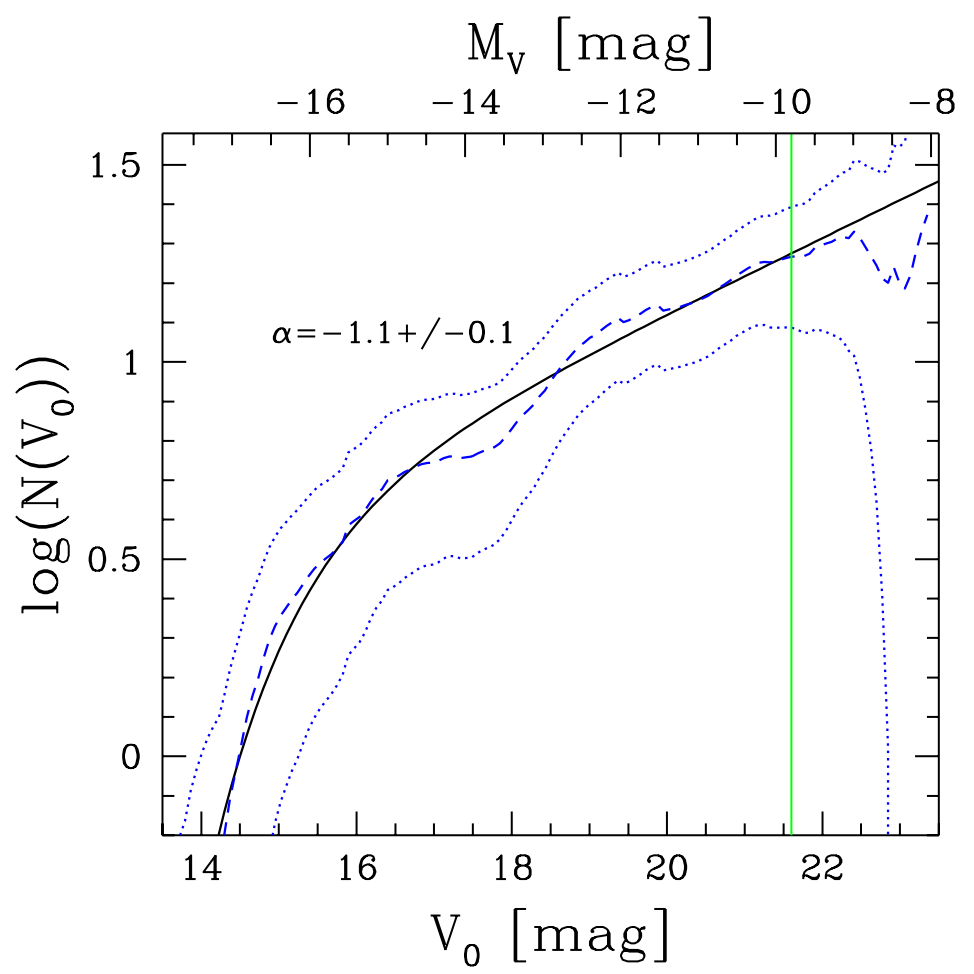

Figure 3. Incompleteness corrected luminosity function of the Fornax early-type dwarf sample as shown in Fig. 2. The 50\% photometric completeness limit is indicated by the vertical (green) line at $M_{V} \sim-10 \mathrm{mag}$. The dashed line is a binning-independent representation of the luminosity function, framed by its $1 \sigma$ uncertainty range (dotted lines). The solid line is a fit of a Schechter function, which yields a rather shallow faint end slope of $\alpha \sim-1.1$.

et al. 2003) may be a signpost of these tidal interactions (Bekki et al. 2003, Mieske et al. 2006).

In Fig. 4 we compare the Fornax cluster dwarf GLF with galaxy luminosity functions in other environments: the Local Group, the Virgo cluster, and the Coma cluster. The absolute scale of all four luminosity functions is from within the virial radius. In the left plot, the luminosity functions are normalised by the dynamically derived virial mass of each cluster. Interestingly, the number of dwarfs per unit mass is highest in the lowest mass environment (Local Group). It is lowest in the highest mass environment (Coma). This is consistent with the finding that the M/L ratio of galaxy groups/clusters increases with group/cluster mass (e.g. Eke et al. 2006). Another way of looking at this is that a steep faint end slope does not imply a higher number of dwarfs relative to the host cluster mass, rather the opposite. In the right plot of Fig. 4, we normalise the luminosity functions by mass density of its host environments. In this representation, environments with a steeper faint end slope have produced more dwarf galaxies.

We conclude that the SBF method is a very powerful tool to help constrain the faint end of the galaxy luminosity function in nearby galaxy clusters. For the Fornax cluster, morphological cluster memberships - if performed at sufficient resolution - are very reliable. 

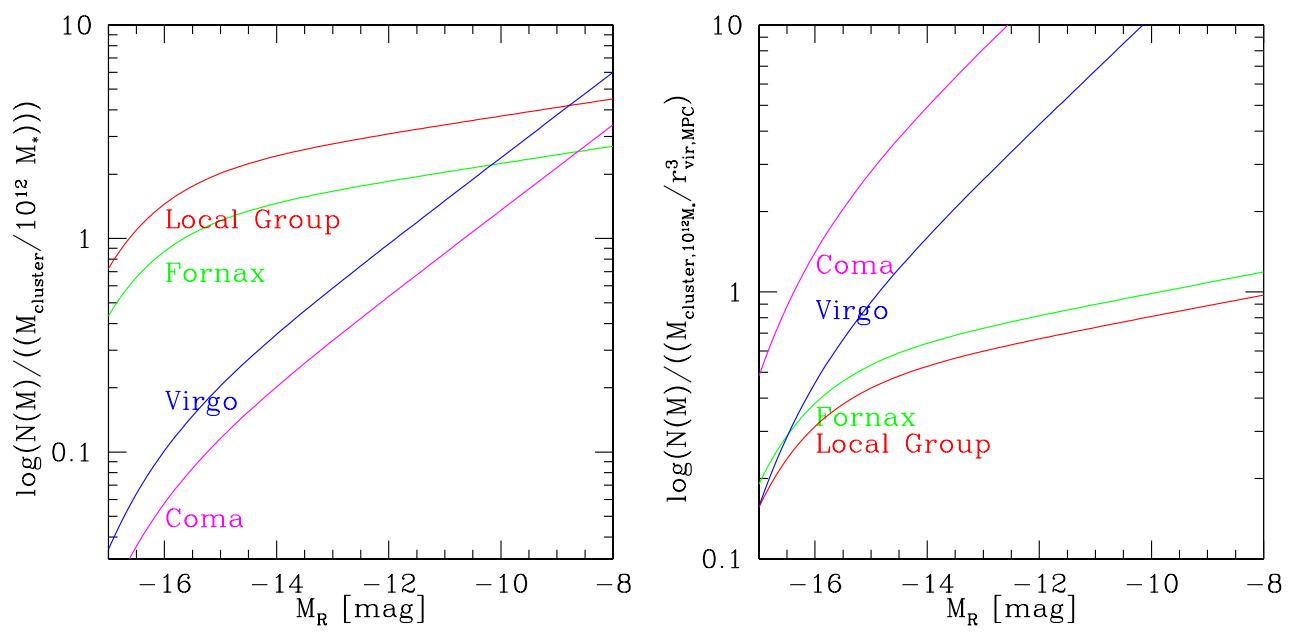

Figure 4. Left: Dwarf galaxy luminosity functions within the virial radii for four different environments (Local Group, Fornax, Virgo, Coma), normalised by the virial mass of each environment. Right: Same luminosity functions as left, but normalised by the mass density within the virial radius. References: Drinkwater et al. (2001), Trentham et al. (2005), Mamon et al. (2004), Sandage et al. (1985), Ferguson \& Sandage (1988), Mieske et al. (2007), Tonry et al. (2000), Mobasher et al. (2003) and references therein, Lokas \& Mamon (2004), Sabatini et al. (2003).

\section{References}

Andreon, S., Cuillandre, J. C., Puddu, E., \& Mellier, Y. 2006, MNRAS, 372, 60

Bekki, K., Couch, W. J., Drinkwater, M. J., \& Shioya, Y. 2003, MNRAS, 344, 399

Côté, P., Marzke, R. O., \& West, M. J. 1998, ApJ, 501, 554

Drinkwater, M. J., Gregg M. D., Holman B. A., \& Brown M. J. I. 2001, MNRAS 326, 1076

Drinkwater, M. J., Gregg, M. D., Hilker, M., et al. 2003, Nature, 423, 519

Eke, V. R., et al. 2006, MNRAS, 370, 1147

Ferguson, H. C., \& Sandage, A. 1988, AJ, 96, 1520

Grebel, E. K., Gallagher, J. S., \& Harbeck, D. 2003, AJ, 125, 1926

Hilker, M., Infante, L., Vieira, G., Kissler-Patig, M., \& Richtler, T. 1999a, A\&AS, 134, 75

Hilker, M., Infante, L., \& Richtler, T. 1999b, A\&AS, 138, 55

Hilker, M., Mieske, S., \& Infante, L. 2003, A\&AL, 397, L9

Infante, L., Mieske, S., \& Hilker, M. 2003, Ap\&SS, 285, 87

Kauffmann, G., \& Haehnelt, M. 2000, MNRAS 311, 576

Jenkins, A., Frenk, C. S., White, S. D. M. et al. 2001, MNRAS, 321, 372

Kambas, A., Davies, J. I., Smith, R. M., Bianchi, \& S., Haynes, J. A. 2000, AJ 120, 1316

Lokas, E. L., Bode, P., \& Hoffman, Y. 2004, MNRAS, 349, 595

Mamon, G. A., Sanchis, T., Salvador-Solé, E., \& Solanes, J. M. 2004, A\&A, 414, 445

Mieske, S., Hilker, M., Infante, L., \& Jordán, A. 2006, AJ, 131, 2442

Mieske, S., Hilker, M., Infante, L., \& Mendes de Oliveira, C. 2007, A\&A in press, astro$\mathrm{ph} / 0706.2724$

Mobasher, B., et al. 2003, ApJ, 587, 605

Moore, B., Ghigna, S., Governato, F. et al. 1999, ApJL, 524, 19

Sabatini, S., et al. 2003, MNRAS, 341, 981

Sandage, A., Binggeli, B., \& Tammann, G. A. 1985, AJ, 90, 1759

Tanaka, M. et al. 2005, MNRAS, 362, 268

Tonry, J. L., \& Schneider, D. P. 1988, AJ, 96, 807

Tonry, J. L., Blakeslee, J. P., Ajhar, E. A., \& Dressler, A. 2000, ApJ, 530, 625

Trentham, N., \& Tully, R. B. 2002, MNRAS 335, 712

Trentham, N., Sampson, L., \& Banerji, M. 2005, MNRAS, 357, 783 